\title{
Local Repair for Extending Lifetime of Wireless Sensor Networks
}

\author{
M. Rai, Shekhar Verma, G.S. Tomar \& Nittin Garg
}

\begin{abstract}
The lifetime of battery operated wireless sensor networks can be prolonged by energy aware maintenance of the virtual backbone. The energy of a node is depleted quickly during communication and network failure is most likely to occur when the energy of a Minimum Connected Dominating Set (MCDS) node is exhausted. The reconstruction of an alternate backbone without the depleted node is both time and energy intensive. In the present study, local repair technique based on neighborhood information has been proposed and evaluated for extending lifetime. An energy depleted node is deleted and the backbone is repaired with other nodes to allow communication. It has been shown that the number of such extra nodes required for repairing the backbone is limited to five. Simulation results shows that the network lifetime extends significantly especially in large dense networks when the local repair is adopted as compared to networks without repair.
\end{abstract}

Index Terms - Backbone, dense network, local repair, minimum connected dominating set

\section{INTRODUCTION}

The lifetime of a wireless sensor networks is its ability to sustain communication between its nodes [1]. In such networks, the messages are usually carried over the virtual backbone and the lifetime of the nodes constituting the backbone determine the life of the network. The lifetime of a wireless sensor network is the time for which the communication is supported till the backbone fails and is irreparable. This happens due to the failure of a backbone node that is irreplaceable. Extending the life of such nodes that participate in the formation of the backbone is the key to prolonging the lifetime of the overall wireless network. The aim is to maximize the life of individual nodes and not the average energy consumption of the network as a whole. The lifetime of a node is the duration for which the power source in the node is able to sustain itself. Since the battery cannot be replaced during the life of the network or a node, only its energy can be conserved by efficient usage.

A sensor network forms an ad hoc, self organizing network [2] [3] [4]. The initialization of a network involves discovery of the neighbors, formation of an energy efficient

Manuscript received Mar, 2009. This work was result of research work going on in the department and supported by project funding agencies and the parent institutes.

Mritunjay Rai is research scholar at ABV-Indian Institute of Information Technology and Management, Gwalior 474010 India.

Shekhar verma, Associated Professor, is with department of Computer Science and Engineering at Indian Institute of Information Technology, Allahabad, 211011 India.

Geetam Singh Tomar is with the Vikrant Institute of Technology \& management, Indore 453331 India as Director of the Institute. topology and the virtual backbone. The principal function of the network is to carry sensor traffic. The traffic per node is intermittent and occurs with very low probability. Energy conservation can, thus, be effected by using energy efficient protocols, scheduling the activity of a node such that it is in the sleep state most of the time or through topology control of the network so that the energy required for broadcast is optimal.

A virtual backbone in a wireless sensor network reduces the communication overhead, increases the bandwidth efficiency, decreases the overall energy consumption and thus increases network operational life. The nodes themselves act as routers in this infrastructure less network. Such a network can be modeled as a unit disk graph, where vertices represent hosts and the unit distance corresponds to transmission range of wireless devices. A minimum connected dominating set (MCDS) [5] [6] [7] forms an optimal virtual backbone in such networks and the determination of MCDS in a graph is an NP Hard problem [2]. Different heuristics have been proposed for the MCDS formation [8] [5] [9] [6] [7]. One set of algorithms [10] is based on the idea of creating a dominating set incrementally. The other set of techniques uses initial set as CDS, recursively remove vertices using Steiner tree etc [11]. Other approaches [12] [13] try to construct a MCDS by finding a maximal independent set, which is then expanded to CDS by adding connected vertices i.e. connectors.

In a network in which the topology and the traffic are fixed, network lifetime is the time for communication is possible. Communication is sustained as long as the present backbone is maintainable or an alternate back bone can be constructed. In general, a battery discharge varies directly as the energy consumption. A sensor node in the MCDS carries a lot of traffic and tends to consume energy draining the battery. A partially discharged battery, however, recharges to a certain extent itself if not used [14] [15]. Thus, a node in the backbone has to be excluded from the backbone when its battery discharges below a certain threshold. The exclusion of a node necessitates modification in the MCDS. An algorithm that reconstructs the MCDS from scratch would consume unnecessary battery power and time. In order to optimize network performance and extend the network lifetime, it is desirable to repair the backbone locally and opt for a suboptimal MCDS instead of an ab initio reconstruction of the MCDS. The heuristic should be able to locally repair the MCDS to prolong the network life, albeit at a slightly degraded performance. In such a network, network lifetime is the time to failure of an irreplaceable node and a backbone

Nittin Garg is with ITC india as executive engineer 
must be repaired till this situation is reached. The paper is organized as follows. Section II contains MCDS construction and section III provides about local repair, in section IV algorithm and model, and section $\mathrm{V}$ presents simulation and results.

\section{MCDS CONSTRUCTION}

The proposed algorithm is divided in three phases. In the first phase, dominating set $D$ is constructed, in second phase set of connectors are found which can connect nodes in $D$, with the help of Steiner tree and in the final phase, pruning is done, where numbers of nodes in the MCDS are reduced to make it optimal minimum connected dominating set. A black node is a node which is to be present in the Connected Dominating Set or is a Dominator. A gray node is a dominatee and a blue node is a connector which is to be present in Connected Dominating Set. In following section an algorithm for finding minimum connected dominating set is presented.

The algorithm proceeds in three stages.

\section{Stage I. Construction of Dominating Set}

In this stage a dominating set is constructed which consists of minimum number of nodes. This stage consists of the following steps:

1) An arbitrary number say $i d$ is assigned to each

Node in the graph $G(V, E)$

2) Each node is assigned white color

3) The node $u$ with maximum degree is taken from $\mathrm{G}(V, E)$ and colored as black, i.e. Dominator

4) All the neighbor nodes of the node $u$ are Colored i.e. Dominatee

5) Do step 3-4 till all the nodes in the graph $G(V, E)$ are colored either as black or gray.

1) Algorithm Stage-I: Algorithm for this stage is given below $\operatorname{STAGE-I}(G(V, E))$

$1 \quad D \leftarrow \varnothing$

2 if $(\forall x \in V$ x.color is black or gray)

\section{3 then exit}

4

5 else $u \leftarrow$ MAX-DEGREE $(V)$

$6 \quad$ u.color $\leftarrow$ black

$$
D \leftarrow D \cup\{\mathrm{u}\} \text { for } \forall x \in N\{u\}
$$

8

do x.color $\leftarrow$ gray

9

10 STAGE-I $(G(V-N[u]))$

2) Explanation of Algorithm Stage-I: Each node in the graph is assigned an arbitrary number as $i d$. Each node is

assigned with white color in the beginning. A node $x \in G(V$,

E) s.t. $x$ has maximum degree is determined; if two nodes have same degree i.e. maximum then choose a node having minimum $i d$. Let that node be $u$. color node $u$ as black i.e. Dominator and this node is added into list of Dominating Set i.e. $D$.

All the neighbors of node $u$ i.e. Dominee are colored as gray so that they are not considered in dominating set. The same is repeated for remaining uncolored graph till all the nodes get colored.

\section{Stage II. Determination of Connectors}

In this stage, set of connectors $B$ is found such that all the nodes in dominating set $D$ gets connected. Let a black node be a node in $D$ and a blue node represent a node in $B$. a node in $B$ is connected by at most $\mathrm{K}$ nodes in the graph $G(V, E)$. Set of blue nodes with given $D$ could be found using Steiner tree. It is a tree, interconnecting all the nodes in $D$ by adding new nodes between them. The nodes that are in the Steiner tree but not in set $D$ are called Steiner nodes. In the MCDS set, the number of Steiner nodes should be minimum. After this stage CDS is constructed, which will consist of black and blue nodes. Let the constructed CDS be set $F$.

This involves the following steps:

1) Select a gray node which is connected to

Maximum $(K)$ number of black nodes, set

Its color as blue

2) Check whether the Dominating Set $D$

3 ) if $D$ gets connected stop

4) else go to step 1 with $K-1$ number of Black nodes

STAGE-II $(G(V, E))$

$1 \quad B \leftarrow \emptyset$

$$
\begin{array}{ll}
2 & \text { for } i \leftarrow \text { Kto } 2 \\
3 & \text { do } \\
4 & \text { while there exist a node } v \text { which is conn- } \\
& \text { ected to } K \text { adjacent black nodes } \\
5 & \\
6 & \text { do }
\end{array}
$$$$
5
$$ 


$$
B \leftarrow B \cup\{v\}
$$

\section{if $D$ is connected}

then exit

10

\section{Stage III. Pruning}

This is the pruning stage. In this stage, redundant nodes are deleted from the CDS constructed in stage II, to obtain the MCDS. In [7] a rule which is based on pruning algorithm has been proposed and requires large number of iterations. Instead of using these rules following steps are used for pruning:

1) Select a minimum degree node $u$ from $F$

2) check if $N[u]$ is subset of $N[1]$ and $N[2]$ and $\ldots N[n]$ where $i$ belongs to $F-\{u\}$

3 ) if step 2 returns true then remove node $u$ and Goto step 1

4) Otherwise donot remove node $u$ and goto step 1

$\operatorname{STAGE-III}(G(V, E), F)$

$1 \quad u \leftarrow \operatorname{MIN}-\operatorname{DEGREE}(F)$

$2 \quad$ if $N[u] \subset N[1] \cup N[2] \cup . . N[n]$

3 then $F=F-\{u\}$

4 STAGE-III $(G(V, E), F)$

$5 \quad / *$ node $u$ is deleted from CDS*/

6 else $\operatorname{STAGE}-\mathrm{III}(G(V, E), F-\{u\})$

$7 \quad / *$ node $u$ is present in CDS but not

8 to be considered in next iterations $* /$

In this section, we present a constant approximation algorithm for MCDS construction in Wireless Sensor Networks. The proposed approximation algorithm works in three stages. In the first stage of the algorithm, the dominating set of the underlying wireless graph is determined by identifying the maximum degree nodes iteratively to discover the highest cover nodes. The second stage connects the nodes in the dominating set through a Steiner tree and prunes this tree to form the MCDS in third stage. For local repair, rule $k$ [7] is applied find the nodes that can maintain the MCDS.

\section{LOCAL REPAIR}

When an MCDS node $m$ goes to sleep, it informs its neighbors. Each node $v_{j}$ of the neighbor set $N(m)$ informs its neighbor set $N\left(v_{i}\right)$. Thus, all nodes in two hop neighbor set, $N 2(m)$, of node $m$ participate in the local repair of the backbone.

Lemma 1: Given a connected unit disk graph $G=(V, E)$, a non MCDS node $v_{i}$ can be adjacent to a minimum of 1 to a maximum of 11 MCDS nodes.

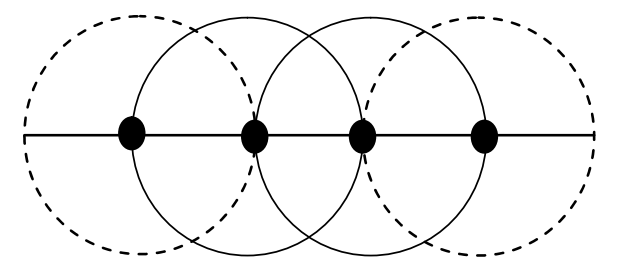

Figure 1. Maximum number of MCDS nodes convering non MCDS nodes

Proof: The proof for the first part the lemma is trivial and follows from the definition of MCDS. It is known that MCDS nodes have to be adjacent. The proof of the second part is as follows, considering a small portion of complete graph, as shown in Figure 2, node $A$ will be surrounded by 6 dependent nodes [13], node $B$ and $F$ are two of them. Node $B$ will dominate node $A$ and node $C$ as both of them are in transmission range of node $B$.

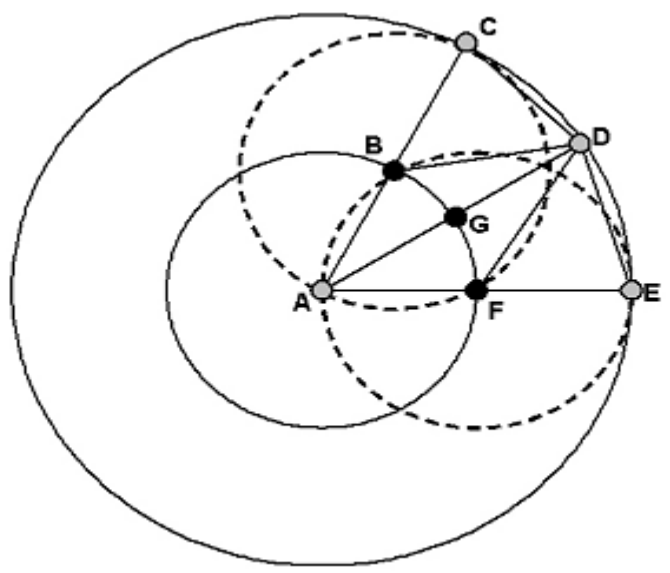

Figure 2. Maximum number of MCDS nodes covering non MCDS nodes

Similarly node $F$ will dominate node $A$ and node $E$. But node $D$ will not be dominated by both node $B$ and node $F$, as distance of node $B$ and node $F$ is more than $d$. Considering

$\triangle A C D$, using cosine rule it is found that $\angle C A D$ is $28.95^{\circ}$ and

$\angle A C D$ is $75.525^{\circ}$, so side $B D$ is $1.225 d$. As distance $B D$ is

more than that of $d$, so node $G$ should also be considered as

dominator. From $\angle C A D$ we know that we can have 12 such

angles at the centre. So $A$ dominatee could be connected to at most 12 MCDS nodes i.e. $K=11$.

Theorem 1: In a unit disk graph $G=(V, E)$, the maximum area that is covered by the union of $n$ MCDS nodes is $(n \pi-2 t)$ 
where $t=(3 / 2+\pi / 3)$ and $\cup A_{i} A_{j}$ all $i, j$ and minimum area is $\pi(\mathrm{r}$ $+1)^{2}$.

Proof: The area coverage by MCDS nodes would be maximized when an MCDS node is connected to minimum number of other MCDS nodes. A terminal MCDS node must be connected to at least one MCDS node, while a non terminal MCDS node would be connected to a minimum of two MCDS nodes. The resulting backbone would form a straight line on the two dimensional Euclidean plane. Figure 1 shows the backbone with MCDS nodes as black nodes with the unit circles as the transmission ranges. From Figure 1, it can be seen that for $\mathrm{n}=1$, the covered area is $\pi$. For $\mathrm{n}>2$ and, the coverage is the area covered by the union of disks which is the sum of areas of the unit disks $(n \pi)$ minus the overlap area $((n-1) t)$. $t$ can be calculated as follows.

Suppose there are 11 MCDS nodes adjacent to one another (Lemma 1). As shown in Figure 2, these 11 MCDS nodes lie on the circumference of a unit circle and dominate at least 11 other non MCDS nodes that lie on a concentric circle of radius 2. Suppose there are other non MCDS nodes that lie on periphery of concentric circles with radii 3 and 4 respectively. Extending the proof of Lemma 1, it can be seen that 23 MCDS nodes are required on the circle of radius 2 to dominate the nodes on the circumference of the circle with radius 3 . Similarly, 47 nodes would be required the 3 unit radius circle to dominate nodes on 4 unit radius circle and so on. This results in the following progression. 11, 23, 47, $95, \ldots t$. The sum of this progression is $12\left(2^{r}-1\right)-r$ where $r$ denote $r_{\text {th }}$ circle.

Hence, given $n$ MCDS nodes, the minimum coverage area would results when $\mathrm{N}<$ sum, and the area would be given by the area of the circle with radius $(\mathrm{r}+1)$ i.e. $\pi(\mathrm{r}+1) 2$.

Theorem 2: The number of additional nodes required to cover the area left uncovered by a deleted MCDS node lies between 2 to 6 .

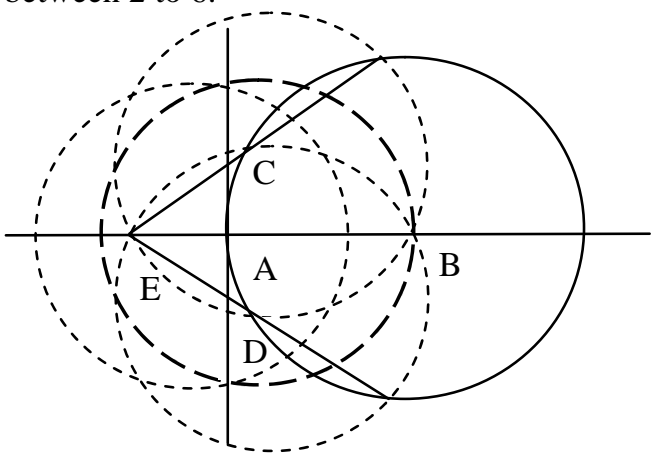

Figure 3. additional nodes for coverage

Proof: Consider Figure 3 that corresponds to the scenario where an MCDS node has maximum coverage. Suppose node $A$ is deleted from the MCDS and additional nodes are required to makeup for the deleted node $A$. The area to be covered, $X=A-B$, is the area not covered by node $B$. Since node $A$ (is an element of MCDS set), the probability that at least one node exists in $X=1$. This entails that at least one extra node is required in the MCDS to cover this node. Now, this node must lie in the area $(A \cap B)$. However, the probability that a node must exist in $(A \cap B)=1$. In this case, the MCDS cannot be repaired. Assume a uniform distribution of the nodes in the unit circles covered by A and B.
The nodes lie on the circumference of the unit circle $B$, farthest from the centre of $B$. The nodes $C$ and $D$ may lie in the neighborhood of $A$ to unit distance away on the intersection of unit circle $A$ and $B$ ( $X$ and $X$ prime). It can be observed that when node $C$ and node $D$ coincide with $X$ and $X$ prime, then an additional node is required at the same place as the deleted node $A$. Since, this is not possible, hence 5 additional nodes are required on the circumference of circle of the deleted node A to cover the area not covered by node B (i.e. $A-B$, the uncovered angular area of $4 \pi / 3$ ). As the points corresponding to node $\mathrm{C}$ and $\mathrm{D}$ move towards point $\mathrm{A}$, only one additional node is required to cover the area $(\mathrm{A}-\mathrm{B})$. When there is only one node (node $\mathrm{C}$ and $\mathrm{D}$ lie on the same location) on the axis joining $A$ and $B$ ), at least two additional nodes are required to cover the area $(A-B)$. Thus, a minimum number of 3 additional MCDS nodes would be required to cover the area uncovered by the deletion of an MCDS node.

\section{MODELS AND ALGORITHMS}

\section{A. Battery Model}

A sensor node acquires data, processes and communicates it. The communication of packets consumes most of the energy of a node. It is assumed that the energy required in transmission and reception is same and same quantity of energy is consumed by all the MCDS nodes in the network backbone [11]. The total energy consumed per node is the energy required per communication and the amount of traffic communicated. The battery power supply in a node depletes by a fixed quantity with each communication. It is known that if a battery is not discharged below a certain threshold, then it recovers some of its charge if rested. The recovery depends on the amount of residual energy in the battery. A sensor node has three different states; active, idle and sleep [13]. When a sensor node is communicating, it is in the active state. When it is ready to communicate, it is in the idle state. A node which is switched off is in the sleep mode. Nodes discharge in the active and idle states; but recover in the sleep mode. In the present study, all nodes not in the MCDS are assumed to be in the sleep state unless they are transmitters or receivers. The MCDS nodes in the backbone are in the idle or active state.

\section{B. Network Lifetime}

Network lifetime is usually defined as the time to first node failure. It is evident that nodes in the backbone are liable to fail earlier than other nodes as they carry all network traffic and their battery power drains with every communication. Hence, network failure is likely to occur due to an MCDS node failure. However, a reconstruction or repair of the backbone can allow communication to be sustained. Even if a backbone cannot be repaired, a communication between two nodes may not need that part of the fractured backbone. Hence, in the present study, network lifetime is taken as the time to the first denial of communication by the network. Obviously, denial of communication implies that the backbone is beyond repair. 


\section{Local Repair Technique}

In Wireless Sensor Networks power consumption is a main issue to be considered. Each node in the Sensor Network get inactivated due to power constraints and low energy level, and due to this node has to be deactivated for restoration. During that period the topology of network changed. To handle this topology change, a new MCDS should be constructed which may be computationally or energy intensive. The Algorithm proposed takes care of node's deletion from the network based on neighboring information.

The issues to be taken care for designing Local repair algorithm are one, deletion of a non-MCDS node will not result in change of MCDS; two, deletion of MCDS node may result in new MCDS for the network.

1) Algorithm: The Local repair algorithm is as follows:

\section{$\operatorname{LOCAL~REPAIR}(G(V, E), d)$}

$1 \quad / * \mathrm{~d}$ is the node to be deleted $* /$

$2 \quad / *$ Let $S$ is the set of node to

3 be consider for Local repair */

$4 S=N(d) \cup$ All the neighboring nodes

5 of MCDS node present in $N(d)$

$6 \quad F=\operatorname{MCDS}-A L G O R I T H M(G(S))$

$7 / *$ MCDS should be constructed in such a way

8 that previous MCDS node remain intact*/

$9 P=\operatorname{STAGE}-\mathrm{III}(G(S), F)$

$10 / * P$ is final MCDS after pruning*/

2) Explanation of Algorithm: Let $G(V, E)$ is the graph representing Wireless Sensor Networks. Let node $v$ gets inactivated from the network. In the proposed algorithm a MCDS is constructed using a set of nodes say $V^{\prime}$ and previous MCDS nodes remains intact i.e. MCDS nodes present before deletion of node $v$. After that both the MCDS are combined to give a complete MCDS. Nodes taken into consideration for Local Repair i.e. set $V^{\prime}$ includes $N(v)$ (open neighboring node of node $v$ ) and neighboring nodes of MCDS node present in $N(v)$.

\section{Illustration}

The above given algorithm can be understood with the help graph shown in figure 3 .

Gray node shows the MCDS Node. Let us consider node 9 is an MCDS node, gets inactivated, resulting graph is shown in Figure 4.

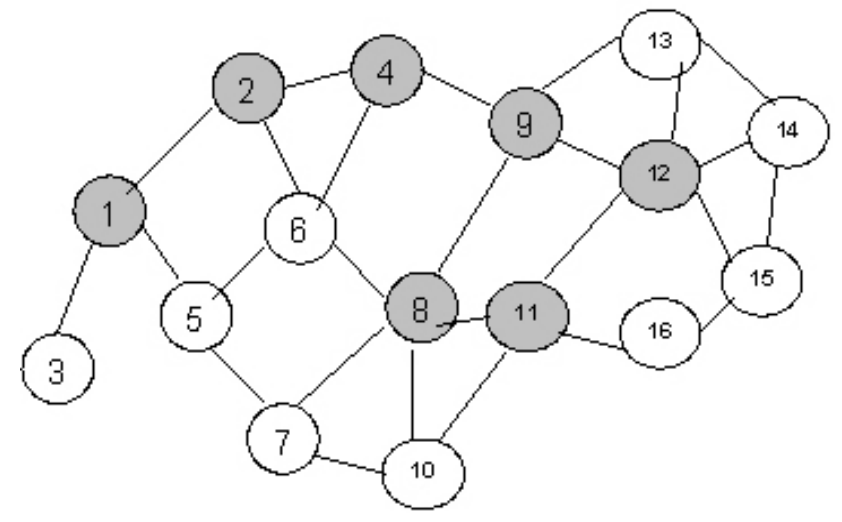

Figure 3. Original Graph

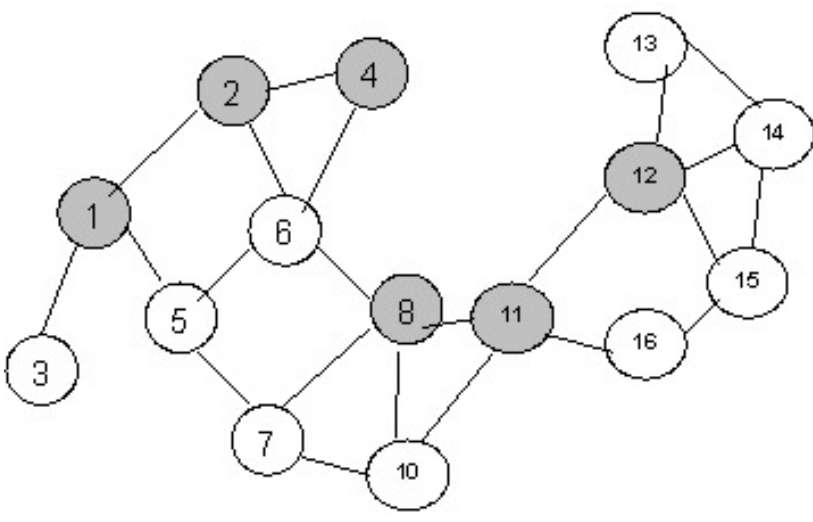

Figure 4. Graph after deleting node 9

For constructing new MCDS for above graph Local repair algorithm is used. Neighbors of inactivated node (node 9) are $N(9)=\{4,8,12,13\}$. These nodes are included in set V'. Apart from that, node 4, 8, and 12 are MCDS nodes, so nodes in $N(4), N(8)$ and $N(12)$ are included in set $\mathrm{V}^{\prime} . \mathrm{V}^{\prime}=\{2$, $4,6,7,8,10,11,12,13,14,15\}$.

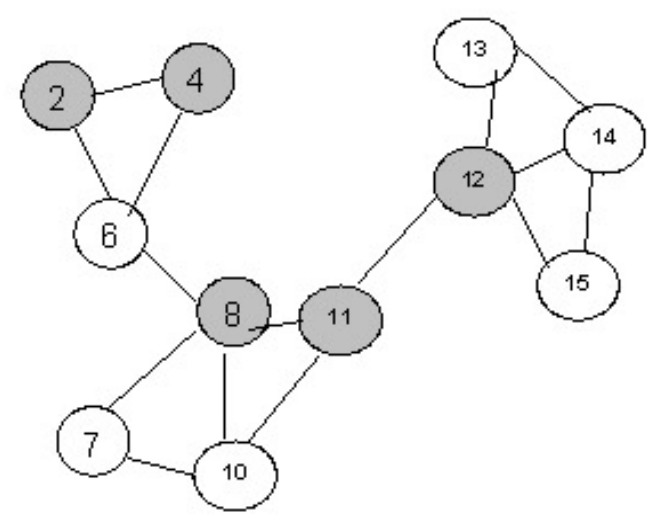

Figure 5. Graph to be considered for Local Repair

Considering the graph shown in Figure 5, a MCDS is constructed such that previous MCDS nodes remain intact. Resulting graph is shown in Figure 6.

CDS of above graph includes nodes $\{2,4,6,8,11$, and $12\}$. Node 6 is newly added MCDS node. Combine this MCDS with previous MCDS result in a graph as shown in Figure 7.

After doing above mentioned steps pruning algorithm may be applied to remove unnecessary nodes from the 


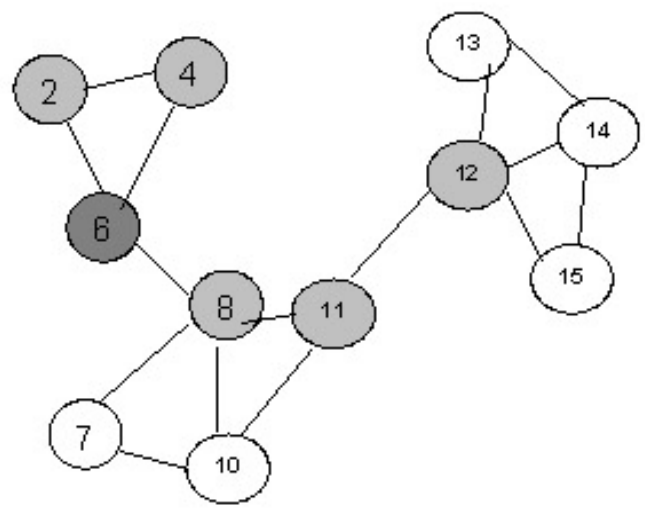

Figure 6. Graph with partial MCDS

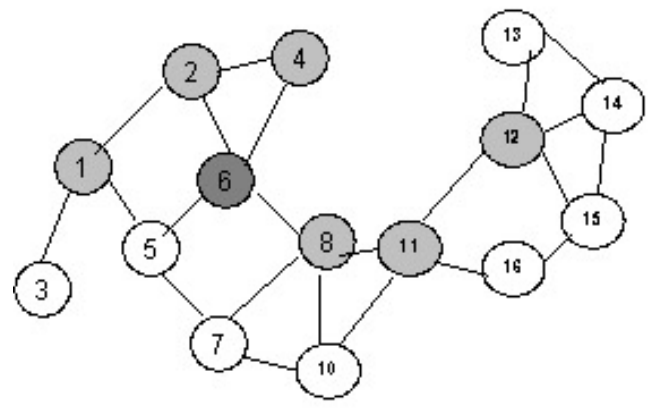

Figure 7. Graph after adding node 6 in MCDS

MCDS. By doing this node 4 gets removed from the MCDS. The resulting graph is shown in Figure 8.

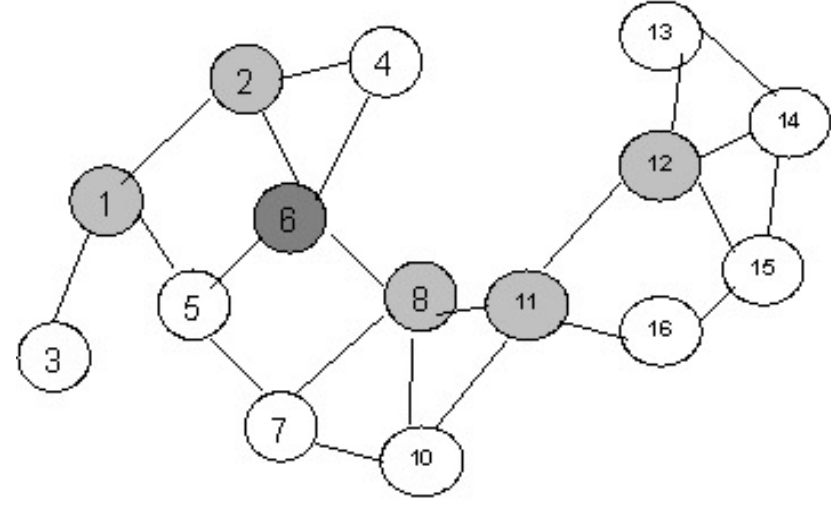

Figure 8. Final Graph after pruning

\section{SIMULATION AND RESULT}

\section{A. Simulation Parameters}

Simulation proceeds in the following manner: In the beginning a network is developed using random number. An adjacency matrix is generated using random number. The density of generated graph varied from sparse to dense. MCDS is then found for these generated graphs using Steiner based heuristic. The density of sparse is considered to be 0.2 , that of medium graph is 0.5 and that of dense graph is 0.8 . The results are simulated for the graph consisting 25 nodes to 350 nodes.

For the purpose of simulation, it is assumed that in the initial construction of the MCDS; all nodes in the network have equal participation and have equal discharge. This energy state of the battery is assumed as the original state.
Once the backbone is constructed, a node may be in one of its three states. In the active state, a node is involved in transmission of messages from source and destination. The battery loses $10 \%$ of its original power in this state. A node moves to sleep state when it is discharged to $50 \%$ of it battery power. In idle state, the node does not transmit or receive any message but node is ready to transmit or receive messages. The node listens for communication requests and if a request arrives, the node switches to the active state. The battery loses $1 \%$ of its current power in this state. In the sleep state a node gets disconnected from the network and cannot receive and transmit messages. The battery recovers in this state. The rate of regeneration is taken to be $10 \%$ of current power and it regenerates till it gets charged to $150 \%$ of current energy. It then moves to the idle state. A node switches to sleep state when it losses $50 \%$ of its original power.

For each run of the simulation, a source $S$ and destination $D$ are selected, and then a minimum hop path containing MCDS between the source and the destination is selected through a shortest path algorithm. All the nodes involved in the path switch to active state and each active node losses its battery power by $10 \%$ of its original power. During this period, active nodes discharge at $10 \%$ of the original energy and idle nodes discharge their battery by $1 \%$ of their present energy. On the other hand, sleeping nodes regenerate. If a node discharges its battery to $50 \%$ of its original, then it moves to sleep state. This triggers the revival of the backbone through local repair of the MCDS. When any node is able to recharge to $75 \%$ of its original energy, it can move to the idle state. This simulation is executed for a network till first failure occur i.e. first request that could not be served either due to involvement of sleep node in the required path or either a terminus node is deleted from the graph or either due to disconnection of the graph. This failure occurs after local repair is no longer possible.

\section{B. Results}

Simulation with the local repair algorithm has been performed and results compared for three types of networks i.e. sparse, medium and dense networks. The simulation was run ten times for each network instance of the network; i.e. for a sparse network with number nodes varying from 25 to 350 ; each instance of the network was simulated ten times. The parameters studied are the initial size of the backbone (number of MCDS nodes), change in the size of the backbone (number of MCDS nodes) due to local repair, time to first repair defined as the activation time, the number of repairs before a node becomes irreparable and the network lifetime.

Figure 4 depicts the average number of MCDS nodes (backbone size) in the different networks before the first repair. It is observed that in sparse graphs, the number of nodes in the backbone becomes almost equal to the total number of nodes as the size of the graph increases beyond 150 nodes. As the density of graph increases, the number of MCDS nodes decreases. This is expected because the increase in density increases the connectivity, thereby, reducing MCDS nodes. 


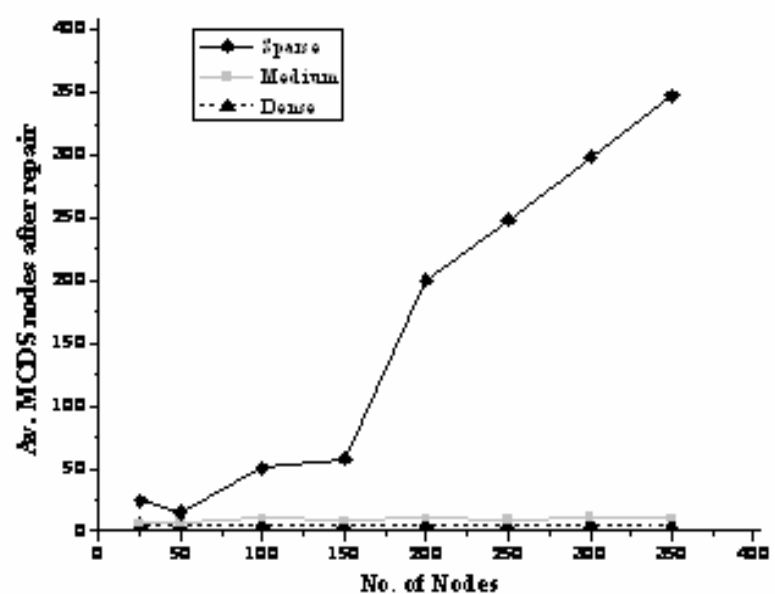

Figure 4. Average numbor of MCDS nodes before repair

It is observed that, as expected, repair increases the number of nodes in the backbone for all types and sizes of the network due to the formation of suboptimal MCDS, as shown in Figure 5. The average increase in higher for sparse and medium graphs as compared to dense graphs with larger number of nodes. This is in consonance with theorem 2 as the average coverage area of each MCDS node in large dense graph is small and number of additional MCDS nodes required to makeup for the deleted node is less a compared to sparse or medium graphs. Moreover, the probability of alternate MCDS node in large and dense graphs is higher in comparison to other graphs.

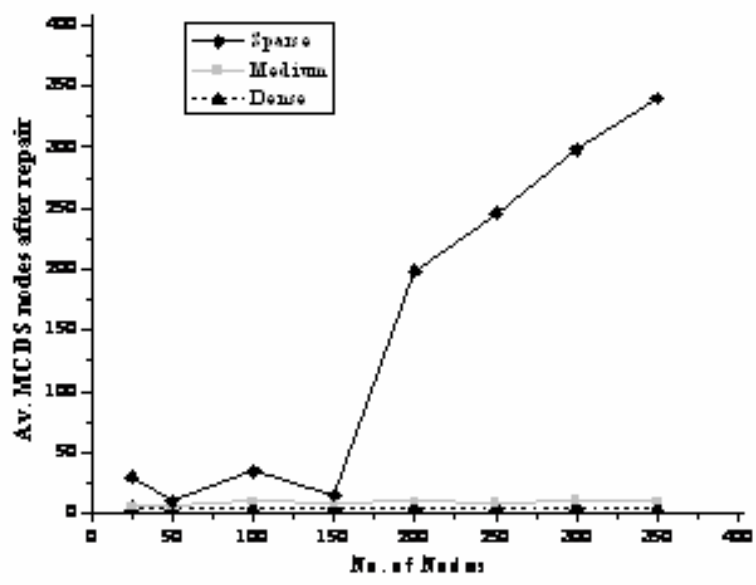

Figure 5. Average number of MCDS nodes after repairs

After this, the network lifetime has been determined for different networks, as shown in Fig. 6. It is shown that for sparse graphs the network lifetime is very small; it is so because as the connectivity of sparse graph would be very low and very less repair possibilities would be available. On the other hand for dense and medium graph it is exactly the reverse. It is observed that the network lifetime for sparse graphs is low and does not increase with the increase in the number of nodes; whereas, the network lifetime increases quite sharply for medium and dense graphs when the number of nodes increased beyond 150 nodes.

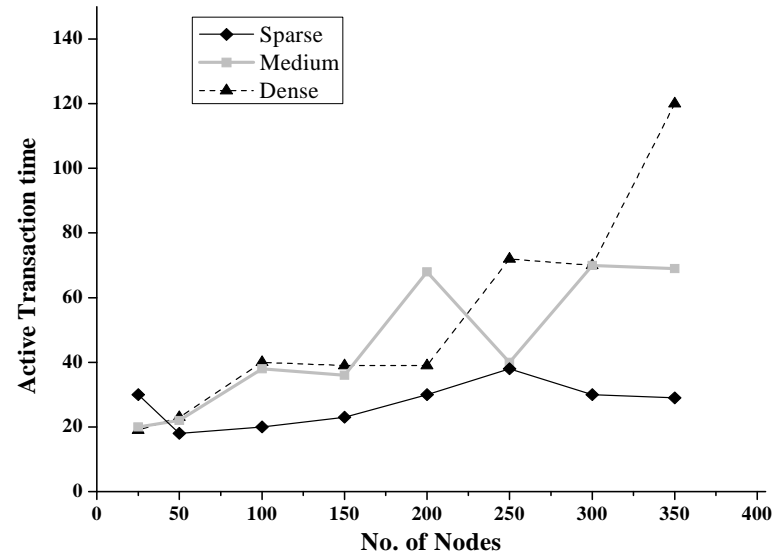

Figure 6. Network Life time.

The activation time is the time for which the network is active without the first repair algorithm. Activation time for sparse graph is less as compared to medium and dense graphs, as shown in Figure 7. It is so because for dense graph the possibility of generating same source is reduced and due to which the simulation could run for longer period but the same is not possible in sparse graphs.

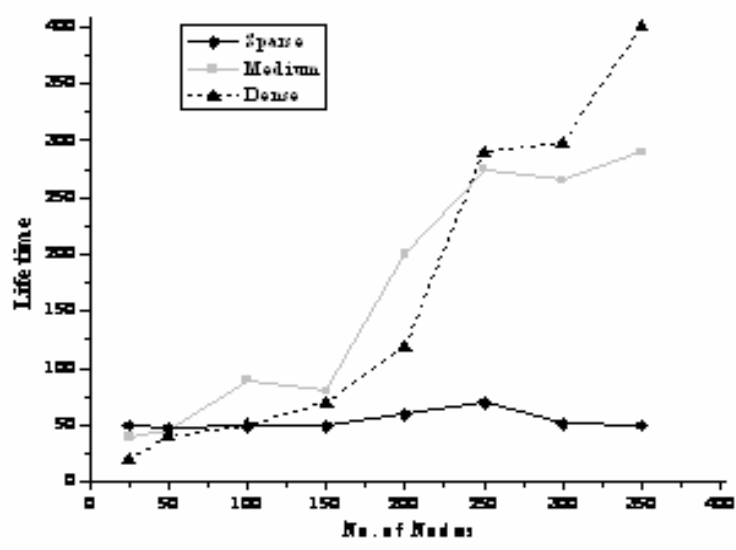

Figure 7. Activation time

In dense graphs, the average number of repairs before the network failure is very less as compare to sparse graphs, as shown in Figure 8. It is observed that network failure occurs in dense graphs due to the irreparability of large number of terminal nodes in dense graphs.

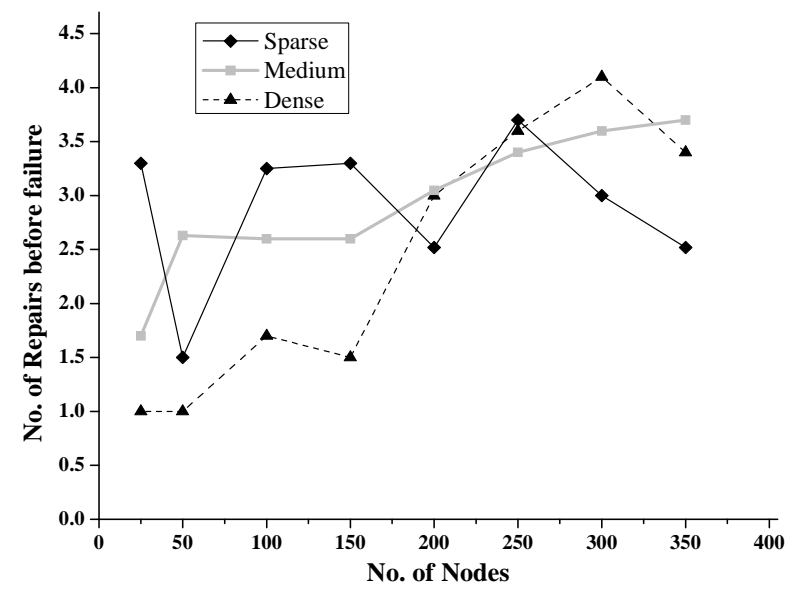

Figure 8. Number of repairs before network failure 


\section{CONCLUSION}

The lifetime of a wireless sensor network in operation is the lifetime of its backbone. The construction of the backbone is both energy and time intensive and an ab initio reconstruction is not desirable. The lifetime of a network is extendible by repair which has to be local. It has been shown that local repair, though, yields a non optimal MCDS, the number of extra MCDS nodes is limited in the worst case to five. Moreover, a local repair technique is able to extend the lifetime of networks significantly especially in large dense graphs.

\section{REFERENCES}

[1] Jae-Hwan Chang, and Leandros Tassiulas, Maximum Lifetime Routing in Wireless Sensor Networks, IEEE/ACM TRANSACTIONSON NETWORKING, VOL. 12, NO. 4, AUGUST 2004.

[2] I. F. Akyildiz, W. Su, Y. Sankarasubramaniam and E. Cayirci, A Survey on Sensor Networks, IEEE Communications Magazine, Aug. 2002, pp. 102-114.

[3] G.J. Pottie, W.J. Kaiser, Wireless Integrated Network Sensors, Communications of the ACM, vol. 43, no. 5, pp. 551-558, May 2000

[4] C. Ma, M. Ma and Y. Yang, Data-Centric Energy- Efficient Scheduling in Densely Deployed Sensor Networks, Proc. of IEEE ICC 2004, Paris, France, 2004.

[5] K. M. Alzoubi, P.J. Wan, and O. Frieder Distributed heuristics for connected dominating set in wireless ad hoc networks. IEEE ComSoc/KICS Journal on Communication Networks, 4(1):22-29, 2002.

[6] B. Das and V. Bharghavan Routing in ad-hoc networks using minimum connected dominating sets. In International Conference on communications, pages 376-380, 1997.

[7] Jie Wu and Hailan Li, On Calculating Connected Dominating Set for Efficient Routing in Ad Hoc Wireless Networks. In Proceedings of the3rd ACM International Workshop on Discrete Algorithms and Methods for Kluwer Academic Publishers, 2004.

[8] S. Butenko, X. Cheng, D.-Z. Du, \& P. M. Pardalos On the construction of virtual backbone for ad hoc wireless network. In editors, Cooperative control: Models, Applications and Algorithms, pages 43-54. Kluwer Academic Publishers, 2002.

[9] I. Stojmenovic, M. Seddigh, and J. Zunic.Dominating sets and neighbor elimination based broadcasting algorithms in wireless networks. In Proc. IEEE Hawaii Int. Conf. on System Sciences, 2001.

[10] Sergiy Butenko, Xiuzhen Cheng, Carlos A. S. Oliveira, P.M. Pardalos A New Heuristic for the Minimum Connected Dominating Set Problem on Ad-Hoc Wireless Networks, 2004. In S. Butenko, R. Murphey, and P. Pardalos, editors, Recent Developments in Cooperative Control and Optimization, pages 6173.

[11] M. Karpinsky and A. Zelikovsky New approximation algorithms for the Steiner tree problem, Technical Report, Electronic Colloquium on Computational Complexity (ECCC): TR95-030, 1995.

[12] Bo Han, HaoHuan Fu, Lidong Lin, and Weijia Efficient Constructionof Connected Dominating Set in Wireless Ad-Hoc Network, IEEE 2004.

[13] T. Thai and Ding-Zhu Du, Connected Dominating Sets in Disk Graphs with bidirectional links, IEEE Communication Letters, Vol-10, No. 3 March 2006.

[14] Chi Ma, Yuanyuan Yang and Zhenghao Zhang Constructing Battery-Aware Virtual Backbones in Sensor Networks, Proceedings IEEE International Conference on Parallel Processing, 2005

[15] Tamaghna Acharya, Rajarshi Roy Distributed Algorithm for Power Aware Minimum Connected Dominating Set for Routing in Wireless Ad Hoc Network, Proceedings IEEE International Conference on Parallel Processing Workshops, 2005

First A. Author and the other authors may include biographies at the end of regular papers. Biographies are often not included in conference-related papers. This author became a Member (M) of IAENG. The first paragraph may contain a place and/or date of birth (list place, then date). Next, the author's educational background is listed. The degrees should be listed with type of degree in what field, which institution, city, state or country, and year degree was earned. The author's major field of study should be lower-cased.

The second paragraph uses the pronoun of the person (he or she) and not the author's last name. It lists military and work experience, including summer and fellowship jobs. Job titles are capitalized. The current job must have a location; previous positions may be listed without one. Information concerning previous publications may be included. Try not to list more than three books or published articles. The format for listing publishers of a book within the biography is: title of book (city, state: publisher name, year) similar to a reference. Current and previous research interests ends the paragraph.

The third paragraph begins with the author's title and last name (e.g., Dr. Smith, Prof. Jones, Mr. Kajor, Ms. Hunter). List any memberships in professional societies other than the IAENG. Finally, list any awards and work for committees and publications. If a photograph is provided, the biography will be indented around it. The photograph is placed at the top left of the biography. Personal hobbies will be deleted from the biography. 